\title{
Experimental and modelling characterisation of residual stresses in cylindrical samples of rapidly cooled bulk metallic glass
}

\author{
Alexander M. Korsunsky ${ }^{1^{*}}$, Tan Sui ${ }^{1}$, Enrico Salvati ${ }^{1}$, Easo P. George ${ }^{2}$, Marco \\ Sebastiani ${ }^{2}$ \\ ${ }^{1}$ Multi-Beam Laboratory for Engineering Microscopy (MBLEM), Department of \\ Engineering Science, University of Oxford, Parks Road, Oxford OX1 3PJ, United \\ Kingdom \\ 2 Institut für Werkstoffe, Ruhr-Universität Bochum, Universitätsstr. 150, 44801 \\ Bochum, Germany \\ ${ }^{3}$ Engineering Department, Roma Tré University, via della Vasca Navale 79, 00146 \\ Rome, Italy
}

\section{Corresponding author:}

Alexander M. Korsunsky

Department of Engineering Science

University of Oxford

Parks Road

Oxford OX1 3PJ

United Kingdom

Tel: +44-18652-73043

Fax: +44-18652-73010

E-Mail: alexander.korsunsky@eng.ox.ac.uk 


\begin{abstract}
Quench processing is widely used in industry to impart the desired structural and mechanical properties by controlling microstructure and compositional gradients, e.g. to obtain supersaturated solid solutions in aluminium alloys, or to achieve martensitic hardening in steels. Rapid cooling, also referred to as quenching or tempering, is also the principal production route for bulk metallic glasses that exhibit high hardness and strength due to their amorphous structure that precludes plastic deformation by easy crystal slip. Importantly, rapid cooling is accompanied by the creation of residual stresses that also have a strong effect on the deformation behaviour.

The present study aims to obtain insight into the residual stresses in cylindrical samples of Zr-based Bulk Metallic Glass (BMG) by combining analytical modelling of thermal and mechanical problems with experimental measurements using Focused Ion Beam - Digital Image Correlation (FIB-DIC) ring-core milling. The results show good agreement between the two approaches, providing improved confidence in the validity of the two approaches considered here.
\end{abstract}

Keywords: Residual stress, bulk metallic glass, quenching, analytical modelling, FIB-DIC ring-core milling, eigenstrain 
Graphical Abstract

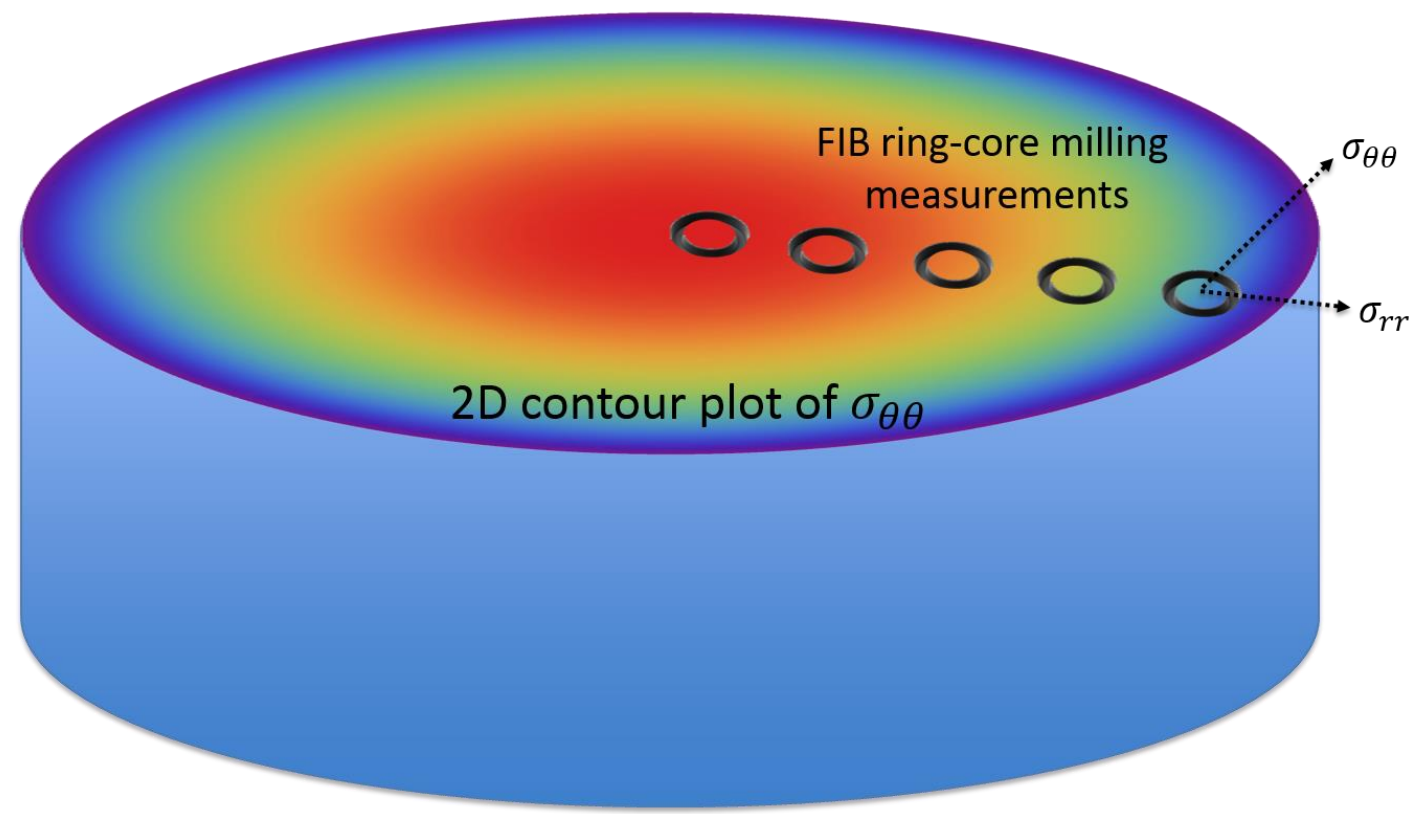




\section{Introduction}

The present short note contains the derivation of the family of explicit closed form solutions in the theory of thermal diffusivity and theory of elasticity that describe the stress states associated with transient problems in heat conduction and stress analysis. Problems of this kind often arise in the studies of materials, e.g. when considering quenching of structural components as part of primary manufacturing or heat treatment, or in the context of the analysis of internal stresses and damage in lithium ion battery electrodes [1].

The study of residual stresses arising as a consequence of thermo-mechanical processing has a long history that goes back to the $18^{\text {th }}$ century work of Gabriel Lamé on the internal stresses in hollow and composite cylinders [2]. The most straightforward case of residual stress generated in a composite cylinder due to the mismatch in the coefficient of thermal expansion can be treated within the framework of elasticity. A circular dissimilar inclusion represents the simplest case of an ellipsoidal inclusion that was later generalised by Jock Eshelby in his famous general treatment presented almost a century later [3].

A series of treatments that combine Lamé axisymmetric treatment with plastic yielding appeared in the second half of the $20^{\text {th }}$ century, e.g. [4]. These approaches allow the prediction of residual stresses that arise as a consequence of yielding under reversed thermo-mechanical loading. Inverse problem formulations have also been proposed aimed at extracting material yield strength from the measurement of residual stresses [5].

A problem of particular interest in materials processing concerns rapid cooling (quenching) that is a heat treatment often necessary to control material microstructure and residual stress, e.g. in glass tempering operations. In the present study we present a combined theoretical and experimental evaluation of the residual stresses within rapidly cooled bulk metallic glass (BMG) cylinders. Bulk metallic glasses possess an unusual combination of properties that makes them attractive for a range of applications. As with many other glassy materials, BMG's can fail by brittle fracture [6]. Therefore, the residual stress state in samples of BMG plays an important contributing role in determining their structural integrity [7]. The purpose of this study is to chart the way towards reliable evaluation of residual stresses in BMG samples at the micro-scale resolution. 


\section{Theory}

The construction of the solution for residual stress in a rapidly cooled glass cylinder consists of two sequential steps. First step is the solution of the transient thermal conduction problem that allows the determination of inelastic strains (eigenstrains) 'frozen in' at this stage. The eigenstrain distribution obtained from the thermal problem solution is then incorporated into the continuum mechanics equations to derive the final residual stress state.

\subsection{Power law approximants for transient thermal diffusion within a cylinder}

Consider a cylindrical object of radius $a$ initially maintained at the normalised temperature $\theta=1$ throughout its volume.

At time zero, the temperature is reduced to zero $\theta=0$ at its surface, and maintained at that level afterwards. The time-dependent temperature distribution within the cylinder is governed by the $2 \mathrm{D}$ transient diffusion equation:

$$
\frac{\partial \theta}{\partial t}=\alpha \nabla^{2} \theta
$$

where $\alpha=\kappa / \rho c_{p}$ is thermal diffusivity expressed through the combination of thermal conductivity $\kappa$, density $\rho$ and heat capacity $c_{p}$ of the body. In the axi-symmetric case considered here the equation (1) simplifies to

$$
\frac{1}{\alpha} \frac{\partial \theta}{\partial t}=\frac{1}{r} \frac{\partial}{\partial r}\left(r \frac{\partial \theta}{\partial r}\right)
$$

The solution is sought by the method of separation of variables, assuming

$$
\theta(r, t)=R(r) T(t)
$$

Substitution into (2) gives the variable separable form:

$$
\frac{1}{\alpha} \frac{T^{\prime}}{T}=\frac{R^{\prime \prime}}{R}+\frac{1}{r} \frac{R^{\prime}}{R}=-\lambda^{2}
$$

The time-dependent part of the solution is given by:

$$
T=\exp \left(-\lambda^{2} \alpha t\right),
$$

where the choice of $-\lambda^{2}$ as the constant is justified by the requirement that the temperature must not become infinitely large with time.

The spatially varying part satisfies the equation

$$
r R^{\prime \prime}+R^{\prime}+\lambda^{2} r R=0
$$


and the solution has the form

$$
R(r)=C J_{0}(\lambda r)
$$

Here $J_{0}(\lambda r)$ is the Bessel function of zero order chosen to ensure that temperature remains finite and continuous at $r=0$.

The values of parameter $\lambda$ are found from the requirement that at the boundary $r=a$ the normalised concentration must be zero. Hence

$$
J_{0}\left(\lambda_{n} a\right)=0 \text {, and } \lambda_{n} a \text { are the roots of } J_{0} .
$$

The general solution is assembled in the form

$$
\theta(r, t)=\sum_{n=1}^{\infty} C_{n} J_{0}\left(\lambda_{n} r\right) \exp \left(-\alpha \lambda_{n}^{2} t\right) .
$$

At time $t=0$ the expression in equation (9) must satisfy:

$$
\theta(r, 0)=\sum_{n=1}^{\infty} C_{n} J_{0}\left(\lambda_{n} r\right)=1
$$

Using orthogonality relation for Bessel functions to enforce boundary conditions, and re-assembling, the final solution is found in the form:

$$
\theta(r, t)=2 \sum_{n=1}^{\infty} \frac{J_{0}\left(\lambda_{n} r\right)}{\left(\lambda_{n} a\right) J_{1}\left(\lambda_{n} a\right)} \exp \left(-\alpha \lambda_{n}^{2} t\right)
$$

Introducing notation $\xi_{n}=\lambda_{n} a$, and normalised variables $\rho=r / a, \tau=\alpha t / a^{2}$, the above solution can be re-written in dimensionless form as:

$$
\theta(\rho, \tau)=2 \sum_{n=1}^{\infty} \frac{J_{0}\left(\xi_{n} \rho\right)}{\xi_{n} J_{1}\left(\xi_{n}\right)} \exp \left(-\xi_{n}^{2} \tau\right) .
$$

Consider the temperature profiles corresponding to the solution expressed by equation (12) and illustrated by continuous curves in Fig. 1 for selected values of normalised time $\tau=\alpha t / a^{2}$. Throughout the process of transient diffusion the temperature distribution can be approximated well by a power law function, $\theta(r)=1-(r / a)^{m}$. The suitability of this approximation is supported by the consideration that small inhomogeneities of material and diffusivity are likely to cause concentration variations comparable or in excess of the difference between the full solution (12) and power law approximants. Hence, subsequent elasticity analyses are built on the basis of this power law assumption. 


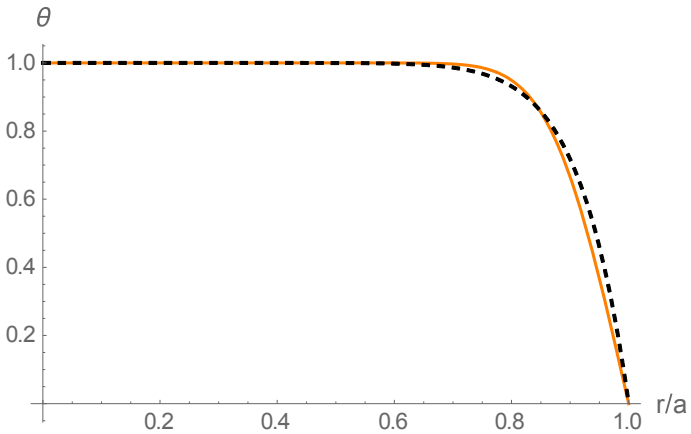

(a)

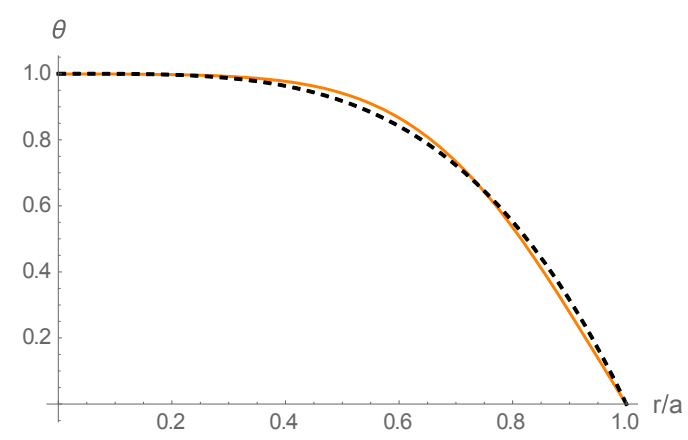

(c)

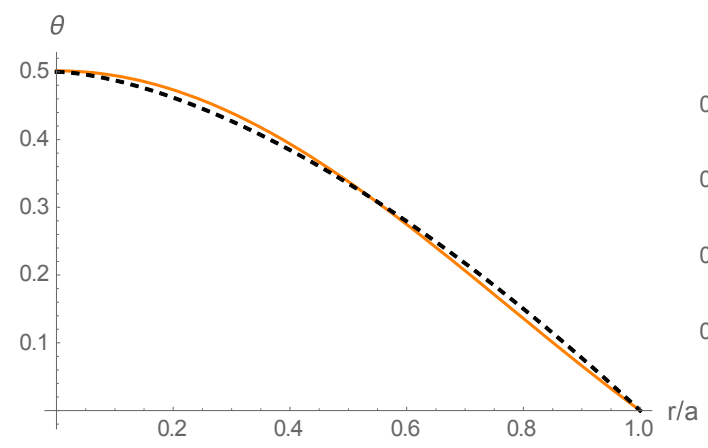

(e)

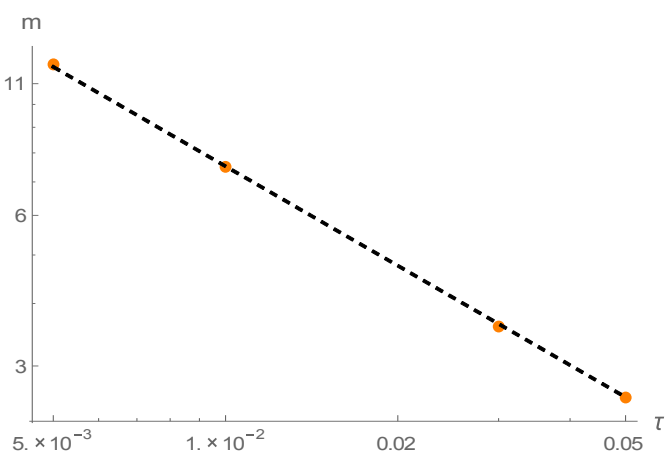

(g)

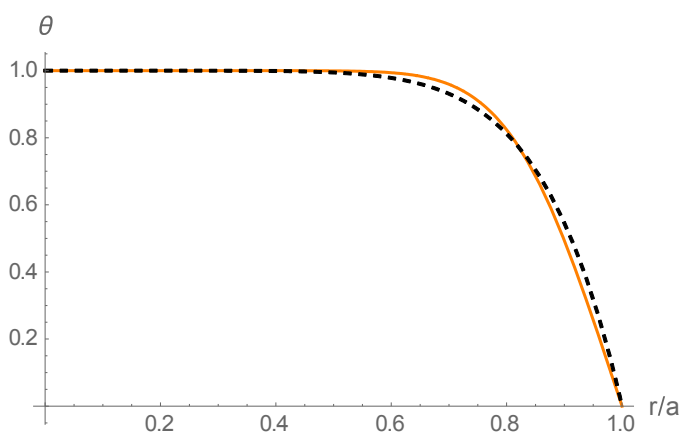

(b)

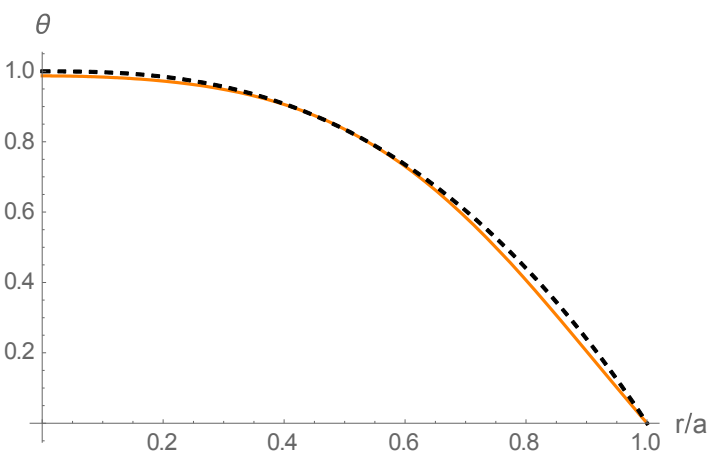

(d)

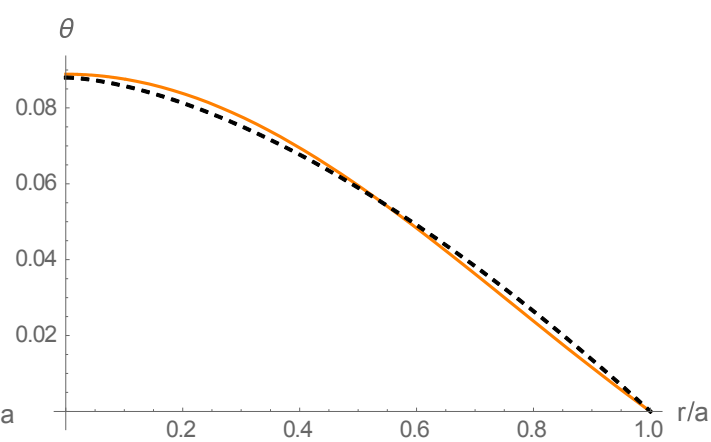

(f)

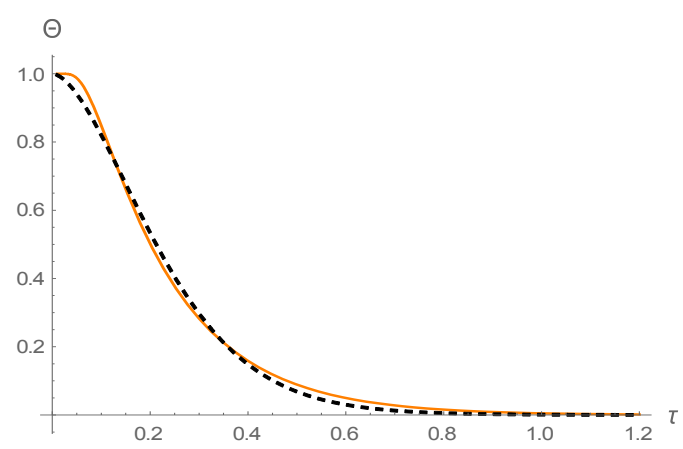

(h)

Fig. 1. Illustration of the closeness of the power law approximation for tempering temperature profile $\theta=1-\rho^{m}$ (black dashed curve) to the solution of the transient diffusion equation (orange solid curve) obtained at different normalised times $\tau$ : (a). $\tau=0.005, m=12$; (b). $\tau=0.01, m=7$; (c). $\tau=0.03, m=3.6$; (d). $\tau=0.05, m=2.6$. (e). Temperature profile fits for the normalised time $\tau=0.2$ and (f) $\tau=0.5$ that are well approximated by the curve 
$\theta=\Theta\left(1-\rho^{m}\right)$, with $m=1.6$ and $\Theta=0.085$. (g). The fit (dashed line) to the power law exponents $m$ in parts (a)-(d) by equation (13). (h). The fit (dashed curve) to the central temperature value $\Theta=\left(1+\tau^{1.7}\right)^{-10}$ that offers a useful approximation for all values of normalised time.

The value of $m$ must be chosen suitably to obtain good agreement. The approximate relationship between the power law parameter $m$ and the normalised diffusion time $\tau$ obtained by least squares fitting illustrated in Fig. 1(g) is given by:

$$
m=0.36 \tau^{-0.66}
$$

In summary, the solution of the transient diffusion equation as a function of time and radial position within the sphere can be expressed to good approximation in the form of the concentration varying as a power law expressed in the form:

$$
\theta(\rho, \tau)=1-\rho^{0.36 \tau^{-0.66}}
$$

The solution persists until the solute concentration at the centre of the sphere begins to differ significantly from the initial value. This occurs at the normalised time of $\tau \cong 0.05$, when $\theta(0)=0.987$, i.e. decreases by $\sim 1.3 \%$.

It is worth highlighting here briefly the significance of formula (14) that presents a closed form, non-series expression for the temperature within a cylinder subjected to cooling (tempering or quenching) as a function of time and coordinate. This form of expression allows ready inversion. For example, the normalised time needed for the temperature at half-radius of the cylinder to be decreased by one tenth of the maximum value is expressed by the compact relation:

$$
0.1=(0.5)^{0.36}
$$

Its inversion gives the following simple result:

$$
\tau \approx 0.0345 \text {. }
$$

For normalised times exceeding $\tau=0.05$, similar approximate descriptions can be elaborated if the following temperature profile is assumed

$$
\theta(\rho, \tau)=\Theta\left(1-\rho^{m}\right)
$$

where $\Theta$ denotes the temperature at the centre. The power law exponent $m$ varies between the value of $\mathbf{2 . 6}$ at $\tau=0.05$ and $\mathbf{1 . 6}$ at $\tau=0.2$, and for practical purposes can be fixed at this value for all $\tau \geq 0.2$. A satisfactory approximation for the central temperature value at all normalised times is given by the 'knee' function [8]:

$$
\Theta=\left(1+\tau^{1.7}\right)^{-10} \text {. }
$$


The above observations demonstrate that expressions (14) and (17) can serve as a useful tool for preliminary design through simple hand calculations. Furthermore, these results allow the solution of the transient thermal diffusion equation to be incorporated in further stress analysis, as shown in the following section.

\subsection{Residual stress in a cylinder containing a radially varying power law eigenstrain}

\section{distribution}

The starting assumption is that cooling causes material contraction that is approximately linearly proportional to the temperature change. Let the maximum thermal strain between the 'hot' and 'cool' states (i.e. initial and final, reduced to the cooling medium temperature) be equal to $\gamma \cong-\alpha \Delta T$. Next, assume that thermal strain causes isotropic expansion or contraction of material, such that the fractional change in each linear dimension is given by strain $\gamma$.

Let us now seek the internal stress solution of the direct problem of eigenstrain theory [9]. Considering a cylindrical body, assume that inelastic strain (eigenstrain) is described by the power law

$$
\varepsilon_{r}^{*}=\varepsilon_{\theta}^{*}=-\gamma(r / a)^{m} .
$$

To determine the stress-strain state that arises within the cylinder, deformation is considered to be axi-symmetric. It is noted that it can be fully described by the radial displacement function $u(r)$. The strain equations take the form:

$$
\begin{aligned}
& \varepsilon_{r}=e_{r}+\varepsilon_{r}^{*}=e_{r}-\gamma(r / a)^{m}=u_{, r}, \\
& \varepsilon_{\theta}=e_{\theta}+\varepsilon_{\theta}^{*}=e_{\theta}-\gamma(r / a)^{m}=u / r .
\end{aligned}
$$

Therefore, the elastic strains are expressed in terms of the displacement function and the thermal strain as follows:

$$
\begin{aligned}
& e_{r}=u_{, r}+\gamma(r / a)^{m}, \\
& e_{\theta}=u / r+\gamma(r / a)^{m}
\end{aligned}
$$

the strains are expressed in terms of stresses as:

$$
\begin{aligned}
& E e_{r}=\sigma_{r}-v \sigma_{\theta}, \\
& E e_{\theta}=\sigma_{\theta}-v \sigma_{r}
\end{aligned}
$$

Introducing the notation 


$$
E^{\prime}=\frac{E}{(1+v)(1-2 v)}
$$

under the plane strain assumption, the stress-strain relations are written as:

$$
\begin{aligned}
& \sigma_{r}=E^{\prime}\left[(1-v) e_{r}+v e_{\theta}\right], \\
& \sigma_{\theta}=E^{\prime}\left[(1-v) e_{\theta}+v e_{r}\right] .
\end{aligned}
$$

Substituting (19) and (22) into the equation of equilibrium [10]:

$$
\frac{\mathrm{d} \sigma_{r}}{\mathrm{~d} r}+\frac{\sigma_{r}-\sigma_{\theta}}{r}=0
$$

we obtain the governing equation for the radial displacement component:

$$
r^{2} u^{\prime \prime}+r u^{\prime}-u=-\gamma m r(r / a)^{m} /(1-v) .
$$

The general solution of this equation has the form

$$
u(r)=A r+B / r-\gamma r(r / a)^{m} /(m+2)(1-v) .
$$

Setting constant $B=0$ ensures that the solution does not have a singularity at $r=0$.

The value of the constant $A$ is found by assuming that the radial stress component vanishes at the particle periphery, $\sigma_{r}(a)=0$.

The elastic strains and stresses are found by back-substitution of this solution into their definition. The stresses have the form:

$$
\begin{aligned}
& \sigma_{\theta}(r)=-\frac{E \gamma}{\left(1-v^{2}\right)} \frac{\left[1-(m+1)(r / a)^{m}\right]}{(m+2)}, \\
& \sigma_{r}(r)=-\frac{E \gamma}{\left(1-v^{2}\right)} \frac{\left[1-(r / a)^{m}\right]}{(m+2)} .
\end{aligned}
$$

Finally, axial stress component can be evaluated using the plane strain assumption

$$
\sigma_{z}(r)=v\left(\sigma_{\theta}(r)+\sigma_{r}(r)\right)=-\frac{E v \gamma}{\left(1-v^{2}\right)} \frac{\left[2-(m+2)(r / a)^{m}\right]}{(m+2)},
$$

It can be readily verified that by construction this expression satisfies the stress balance requirement for the cylindrical body under consideration.

The significance of the outcome of the two sections above is that the solution of the axisymmetric thermo-mechanical problem within a solid cylinder can be obtained in closed form at any stage of cooling, since the temperature distribution at all stages can be represented well by a power law function with parameters calibrated against the solution in the form of series sum of products of Bessel functions describing 
spatial variation and exponents describing time dependence. In the next section this solution is applied to the case of tempered glass cylinder.

\section{3. 'Instant freeze model' evaluation of residual stresses in a tempered glass cylinder}

The prediction of residual stresses in glass components subjected to tempering is a long-standing problem that has been tackled by many researchers. Some of the earliest systematic studies found in the Russian literature [11, 12] have been popularised more recently in the work of Aydiner and Üstundag [13], who applied the analytical expressions derived on the basis of this approach to the problem of tempering a BMG plate.

Indenbom's approach that is also referred to as the instant freeze model is based on the separation of total strain in a visco-elastic material into three additive components, namely: elastic strain, viscous strain, and thermal strain:

$$
\varepsilon^{t o t}=\varepsilon^{e l}+\varepsilon^{v i s}+\varepsilon^{t h} .
$$

Pure elastic stress-strain relationship is assumed. Furthermore, it is also assumed that above its glass transition temperature, the materials does not sustain any elastic strain (and hence stress is zero). Upon cooling to $T_{g}$ instant glass transition vitrification - occurs from the viscous state to the glassy state (hence the model title). At this point the viscous strain component is 'frozen in' permanently, and becomes eigenstrain that acts as the source of residual stress. Since heat flows outwards from the core of the body, vitrification proceeds by the inward advancement of the socalled 'freezing front'. When this freezing front reaches the centre of the body, i.e. when the temperature at the body centre drops to $T_{g}$, no further change in the eigenstrain distribution takes place: the final residual stress state at room temperature arises from the superposition of subsequent cooling on this 'frozen in' state that involves the interplay between changing thermal and elastic strains.

Indenbom's Instant Freeze Model (IFM) originally developed for plate geometry to the case of tempered cylinders in the present study is adapted through the use of two key assumptions: 
(i) that the driver for residual stress generation in the final state of the body is the maximum difference between residual strains (eigenstrains) across the body at the point of final freezing (vitrification) at the core of the cylinder; and

(ii) that the uniformity of total strain at the point of final freezing at the cylinder centre means that the 'frozen in' eigenstrain is equal and opposite to the thermal strain prevailing in the sample at that moment.

Direct application of the above assumptions means that the residual stress state in a tempered glass cylinder can be obtained by selecting the appropriate solution reported in the previous section. A change of sign should be applied, since the 'frozen in' eigenstrain driving the residual stress state is assumed to be equal and opposite to the thermal strain at the point of final freezing.

\section{Experimental}

\subsection{Material and method}

A series of coupons from a bulk metallic glass cylinder were chosen as samples for the present study. The target composition (at.\%) of the bulk metallic glass used in this study was $52.5 \mathrm{Zr}-17.9 \mathrm{Cu}-14.6 \mathrm{Ni}-10 \mathrm{Al}-5 \mathrm{Ti}$.

This bulk metallic glass was made by arc melting high-purity $\mathrm{Zr}, \mathrm{Cu}, \mathrm{Ni}, \mathrm{Al}$, and Ti in pure argon followed by drop-casting into a cylindrical copper mould initially held at room temperature. The diameter of the resulting cylindrical rod was $6.7 \mathrm{~mm}$ and its length was $72 \mathrm{~mm}$. Weight loss after casting was less than $0.1 \%$, implying that the actual composition of the cast rod was close to the aim composition. Specimens were obtained by cutting slices from the original rod, followed by polishing them using an automated procedure consisting of a sequence of grinding steps (from 30 to 5 $\mu \mathrm{m})$, and a final step in which the sample was polished by a cloth containing $0.1 \mu \mathrm{m}$ colloidal silica suspension for 100 hours.

Mechanical properties of the BMG sample adopted in the calculation were drawn from the data for similar material and composition in the literature [12, 14], which gives Young's modulus of $89 \mathrm{GPa}$, Poisson's ratio of 0.37 , and the thermal expansion coefficient that to the first approximation can be assumed to remain constant and equal to $\sim 10 \times 10^{-6}$. The room temperature compressive strength of $\sim 1.8 \mathrm{GPa}$ has been reported [15]. 


\subsection{FIB-DIC ring-core method and residual stress evaluation}

One of the few techniques suitable for residual stress measurement at the microscale in amorphous material is the approach known as FIB-DIC ring-core milling [16]. The method has significant similarities to the well-established macroscopic ring-core method [17], in that the technique relies on the strain relief phenomenon that occurs within the material after a cut is introduced. In the FIB-DIC ring-core method this is performed by Focused Ion Beam milling. The chosen cut geometry (circular trench) isolates a cylindrical volume from the surrounding parent material down to a certain depth, creating a micro-pillar (core). The process is conducted incrementally, with SEM images of the top surface of the micro-pillar acquired in the beginning and at each new depth of cut. The analysis of the pillar top surface deformation during milling is performed using Digital Image Correlation (DIC) that allows the determination displacements, and hence of the strain field.

The key input into subsequent interpretation is the record of strain relief as milling progresses. This data is evaluated by least squares fitting to it a master function [16] that is derived from FEM simulation of the milling process. The outcome of the fitting procedure is the value of complete strain relief that is related to the underlying residual stress via a simple elasticity equation. This is described at length and in considerable detail in [18], and therefore is not repeated here. The uncertainty of stress determination is evaluated by error propagation that includes the errors arising from DIC marker tracking [18], displacement field fitting to determine strain [16], stress calculation [19] and the possible effect of FIB damage [20]. The sample of bulk metallic glass with the diameter of $6.7 \mathrm{~mm}$ was coated with a thin film of $\mathrm{Au}$ a few nanometres thick using an in vacuo sputter coater. FIB ring-core milling was performed with $5 \mu \mathrm{m}$ diameter markers and with the trench width of $1 \mu \mathrm{m}$. After the initial SEM micrograph of the region of interest at the sample surface was taken, a series of 50 milling steps was carried out at the increment of $100 \mathrm{~nm}$, with an SEM image taken after each milling step. A milling current of $133 \mathrm{pA}$ was used to prevent surface damage during the milling process. Five FIB-DIC measurements were made at positions of $0.25 \mathrm{~mm}, 1.00 \mathrm{~mm}, 1.75 \mathrm{~mm}, 2.60 \mathrm{~mm}$ and $3.40 \mathrm{~mm}$ from the edge of the sample. Fig. 2(a) illustrates the location of the FIB-DIC markers at sample surface, and the inset shows a representative SEM image of the milling operation taken in the course of experiment. Fig. 2(b) shows a representative fit of the master curve to the strain relief data as a function of the normalized milling depth. 

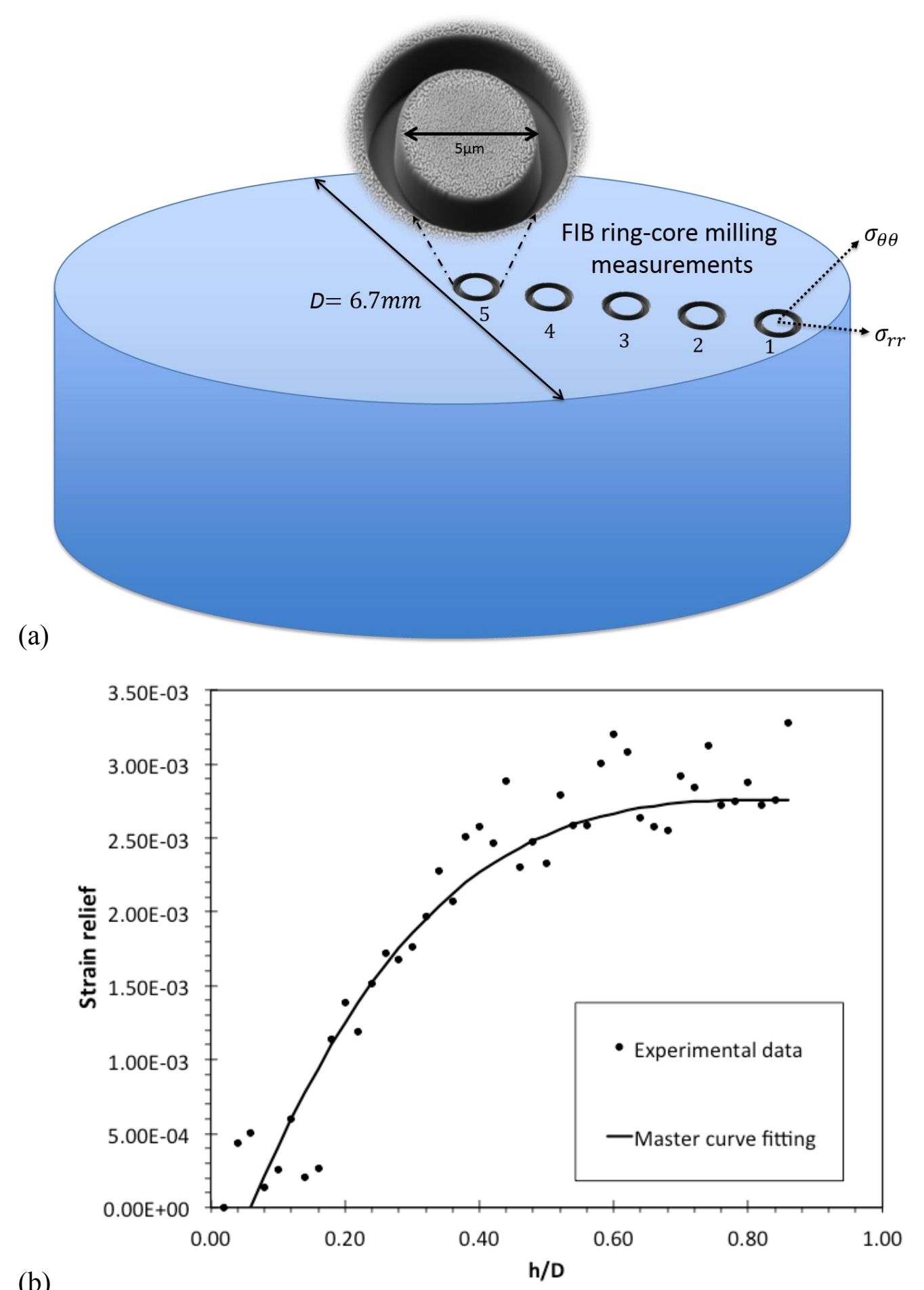

(b)

Fig.2 (a) Illustration of the five milling positions along the radial direction, with the inset showing a representative image of the FIB-DIC marker. (b) Illustration of the master curve fit to experimental strain relief $v s$ depth data to obtain the complete strain change parameter.

The modified DIC Matlab script (the iStress code) based on the original programme written by Chris Eberl [21] was used to perform DIC analysis on the central island. Extra features used in our version included automated master curve 
fitting and error estimation by standard deviation propagation. The textured surface was found to provide sufficient contrast to enable the markers to be tracked effectively, and the errors in the data were found to be small. DIC analysis on the central micro-pillar was conducted for the radial and tangential components of strain.

\section{Comparison of model prediction and experimental measurements}

FIB-DIC ring-core measurements revealed the presence of a variation along the radial direction of both hoop and radial components of residual stress and strain. As illustrated in Fig.3 (a), satisfactory agreement was obtained between the model prediction and experimental data with minimal adjustment of parameters. Fig. 3(b) shows a contour plot representation of the reconstructed residual stress distribution.

The results obtained in this initial study provide a proof of feasibility of the approach, and demonstrate that it warrants further systematic investigation of its flexibility and suitability for the study of the relationship between temperaturedependent mechanical properties and thermal treatment schedule, on the one hand, and the resulting residual stress state within quenched and tempered glass cylinders, on the other.

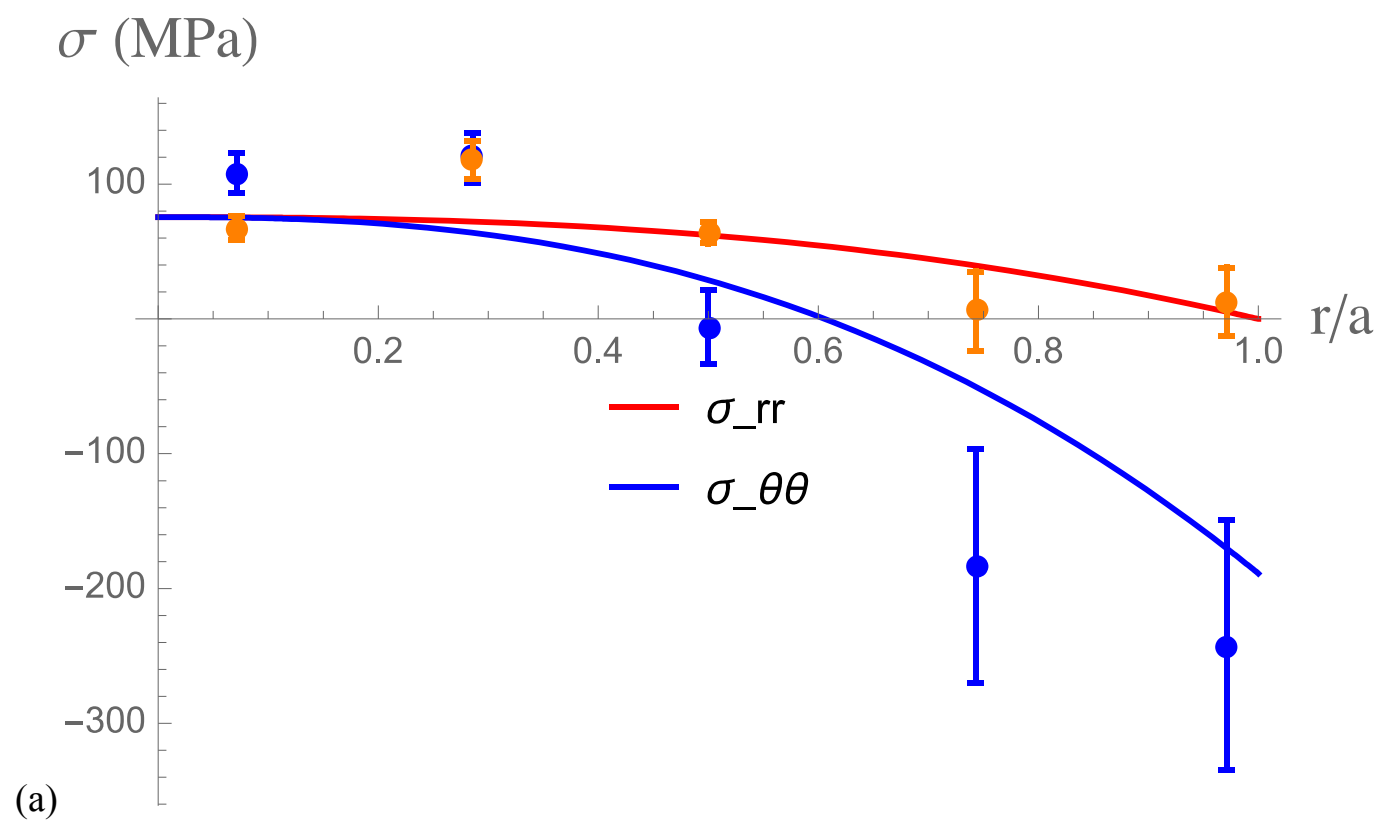


(b)

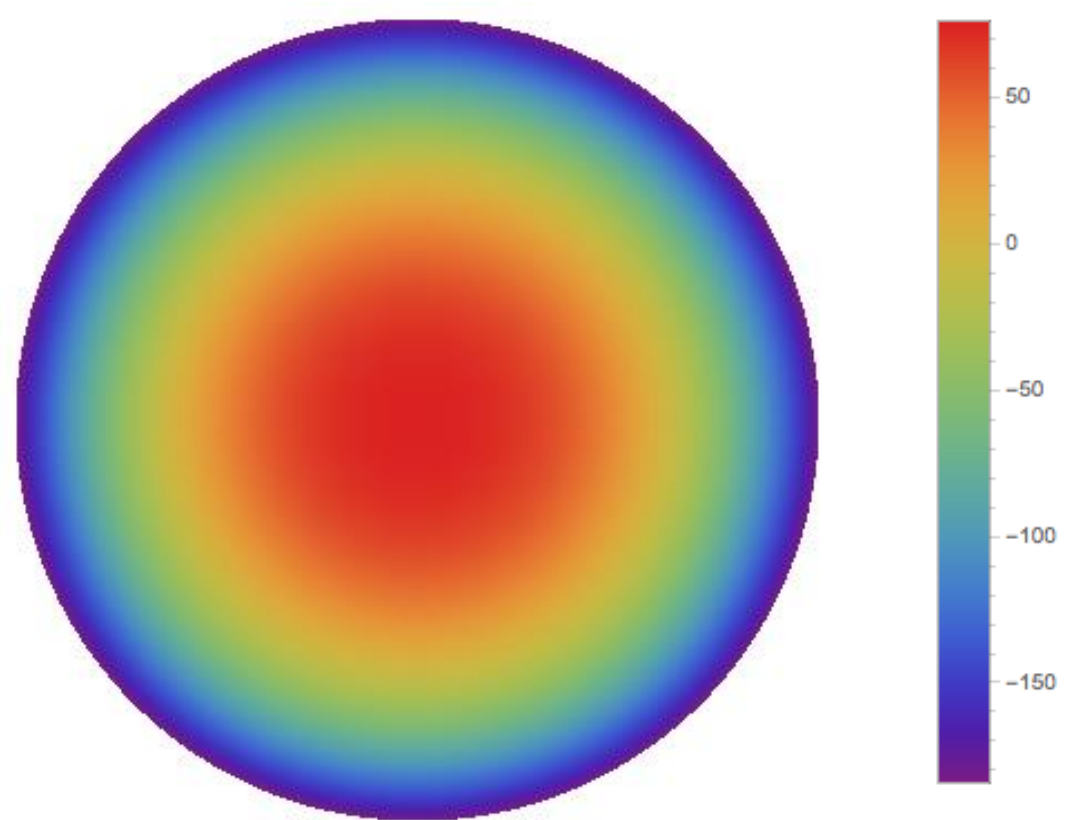

Fig. 3. (a) Radial and hoop stresses predicted by the IFM (continuous curves) and measured by ring-core FIB-DIC. (b) Contour plot representation of the residual stress $\sigma_{\theta \theta}$ in the cylinder. Colour scale is in the units of $\mathrm{MPa}$.

\section{Conclusions}

The analytical treatment presented in this short note establishes the basis for efficient numerical analysis of the internal stresses in rapidly cooled glass cylinders. The expression established for transient cooling of a solid cylinder provides simple power law formulae for the temperature as a function of time and radial coordinate. This expression is then incorporated in the eigenstrain-based solution for the residual stress state, leading to the final closed form formulae for stresses within the tempered glass cylinders. It is worth noting the satisfactory agreement of the model predictions with experimental measurements of residual stress using FIB-DIC micron-scale ringcore milling.

\section{Acknowledgements}

AMK acknowledges funding received for the MBLEM laboratory at Oxford through EU FP7 project iSTRESS (604646), and access to the facilities at the Research Complex at Harwell ( $\mathrm{RCaH})$, under the Centre for In situ Processing Studies (CIPS).

MS acknowledges the funding received from the USA-Italy Fulbright commission, and wishes to thank Prof. George M. Pharr for his support and useful discussions of experimental activities. MS acknowledges access to the interdepartmental laboratory 
of electron microscopy (LIME) at Roma TRE University for performing FIB experiments.

The bulk metallic glass was prepared while EPG was at ORNL under sponsorship of the U.S. Department of Energy, Office of Science, Basic Energy Sciences, Materials Sciences and Technology Division.

\section{References}

1. A.M. Korsunsky, T. Sui, B. Song, Explicit formulae for the internal stress in spherical particles of active material within lithium ion battery cathodes during charging and discharging. Materials and Design, 69, p. 247-252, 2015.

2. G.L.J.B. Lamé, Leçons sur la théorie mathématique de l'élasticité des corps solides. Bachelier, 1852.

3. J.D. Eshelby, The determination of the elastic field of an ellipsoidal inclusion, and related problems, Proceedings of the Royal Society A, 241 (1226), p.376-396, 1957. doi:10.1098/rspa.1957.0133

4. T.B. Kammash, S.A. Murch, T.M. Naghdi, The elastic-plastic cylinder subjected to radially distributed heat source, lateral pressure and axial force with application to nuclear reactor fuel elements, J. Mech. Phys. Solids, 8, p.1-25, 1960.

5. A.M. Korsunsky, Residual elastic strains in autofrettaged tubes: elastic-ideally plastic model analysis, J. Engng. Matls. Technol., 129(1), p.77-81, 2007

6. Y. Jiang, K. Qiu Computational micromechanics analysis of toughening mechanisms of particle-reinforced bulk metallic glass compositesOriginal Research Article, Materials \& Design, 65, p. 410-416, 2015.

7. Y. Huang, H. Fan, D.Wang, et al., The effect of cooling rate on the wear performance of a $\mathrm{ZrCuAlAg}$ bulk metallic glassOriginal Research Article, Materials \& Design, 58, p. 284-289, 2014.

8. R. Busch, Z. Evenson, I. Gallino, S. Wei, Thermodynamics, kinetics and fragility of bulk metallic glass forming liquids, Eprint Arxiv, 304(00), p. 97-102, 2014.

9. A.M. Korsunsky, Eigenstrain analysis of residual strains and stresses, The Journal of Strain Analysis for Engineering Design, 44 (1), p.29-43, 2009.

10. A.F. Bower, Applied Mechanics of Solids, CRC Press, 2011.

11. G.M. Bardenev, Zhurnal Tekhnicheskoi Fiziki, 18, p. 383-388, 1948.

12. V. L. Indenbom, Journal of Technical Physics, 24, p. 925, 1954. 
13. C.C. Aydiner, E. Üstundag, J.C. Hanan, Thermal-tempering analysis of bulk metallic glass plates using an instant-freezing model, Metallurgical and Materials Transactions A, 32(11), p.2709-2715, 2001.

14. Z. Zhang, V. Keppens, P. K. Liaw, Elastic properties of Zr-based bulk metallic glasses studied by resonant ultrasound spectroscopy, J. Mater. Res. 22 (2) p. $364-$ $367,2007$.

15. S. Xie, E. P. George, Size dependent plasticity and fracture of a metallic glass in compression, Intermetallics, 16, p. 485-489, 2008.

16. A.M. Korsunsky, M. Sebastiani, E. Bemporad, Residual stress evaluation at the micrometer scale: Analysis of thin coatings by FIB milling and digital image correlation. Surf Coat Tech, 205(2), p. 393-403, 2010.

17. C. Bouffioux, R. Pesci, R. Boman, N. Caillet, J.-P. Pointhot, A.M.Habraken, Comparison of residual stresses on long rolled propiles measured by X-ray diffraction, ring-core and the sectioning methods and simulated by FE method. Thin-Walled Structures, 104, p. 126-134, 2016.

18. A.J.G Lunt. A.M. Korsunsky. A review of micro-scale focused ion beam milling and digital image correlation analysis for residual stress evaluation and error estimation. Thin Solid Films, 572(1), p. 224-231, 2014.

19. E. Salvati, T. Sui, A. M. Korsunsky. Uncertainty quantification of residual stress evaluation by the FIB-DIC ring-core method due to elastic anisotropy effects. International Journal of Solids and Structures, 87(1), p. 61-69, 2016.

20. E. Salvati, T. Sui, A.J.G. Lunt, A.M. Korsunsky. The effect of eigenstrain induced by ion beam damage on the apparent strain relief in FIB-DIC residual stress evaluation Materials \& Design, 92, p. 649-658, 2016.

21. Digital Image Correlation (DIC) code di-hatJHU, running in Matlab, free download from, http://www.mathworks.com/matlabcentral/fileexchange/12413. 